\title{
Hepatoprotective Effect of S-ademetionine in the treatment of Intrahepatic Cholestasis through Farnesoid X Receptor Mechanism in Ratsp
}

\author{
Mao $\mathrm{H}^{*}$, Jin D, Lu M, Jin S and Huang $\mathrm{Y}$ \\ Department of Gastroenterology, Zhujiang Hospital, Southern Medical University, Guangzhou 510282, China
}

\begin{abstract}
S-ademetionine (AdoMet) had been extensively studied in several clinical studies to treat Intrahepatic Cholestasis (IHC). The exact mechanism of action of AdoMet to regulator bile acid is unknown. Farnesoid X Receptor (FXR), a multifunctional nuclear receptor, plays vital roles in metabolism of bile acid. This study aims to investigate the mechanism of action of S-ademetionine in FXR to treat IHC. The IHC Sprague Dawley (SD) rat model was developed by administering Alpha Naphthyl Isothiocyanate (ANIT, 50 mg/kg). FXR excitant GW4064 (3 mg/kg) was used as a control to observe the impact of AdoMet $(60 \mathrm{mg} / \mathrm{kg})$ on the changes in serum direct levels in these rats. The hepatic histology between the HE staining contrast groups was performed using the Q-PCR to test FXR and the in situ hybridization was used to test the changes of bile salt export pump (Bsep), multidrug resistance-associated protein 2 (Mrp2), and $\mathrm{Na}^{+}$-taurocholate cotransporting polypeptide (Ntcp). AdoMet significantly reduced the serum direct levels and the hepatic tissue damage. AdoMet can also increase the expression of FXR, Bsep, Mrp2 and Ntcp. These findings confirm that AdoMet could reduce serum direct levels as well as improving hepatic tissue damage by enhancing the expression of FXR, Bsep, Mrp2 and Ntcp. These findings could also explain the great therapeutically effect of AdoMet in intrahepatic cholestasis.
\end{abstract}

Keywords: S-ademetionine; Liver; Intrahepatic cholestasis; Farnesoid X receptor

\section{INTRODUCTION}

Cholestasis is a condition where flow of bile from liver to duodenum is obstructed, which results into accumulation of bile acid in liver. Accumulation of toxic bile acid may cause hepatocellular inflammatory reactions leading to liver tissue fibrosis and eventually developing into hepatocirrhosis, liver cancer or cholangiocarcinoma. The Farnesoid X receptor (FXR) is a member of the nuclear receptor superfamily and had gain attention as a critical player in the control of several metabolic pathways [1]. Upon activation by bile acids, FXR regulates bile acid synthesis, conjugation and transport as well as lipid and glucose metabolism. Of the target genes of FXR regulate metabolism of bile acid, Bsep (bile salt export pump) mediates univalent bile acid in hepatic cells to be transported into the bile duct; Mrp2 (multidrug resistance-associated protein 2) mediates non-bilesalt organic anions such as bilirubin, bivalent bile acid, bilirubin glucuronide and glutathione to be excreted into the bile duct; Ntcp ( $\mathrm{Na}^{+}$-taurocholate $\mathrm{co}$ - transporting polypeptide) is the principal bile acid absorption system expressed at the basilar membrane of hepatic cells, and serves as a main transporter which absorbs bile acid from portal vein [2-5]. Activation of FXR may regulate the expression of Bsep, Mrp2 and Ntcp in the liver, which in turn enables hepatic cells and bile capillary to regulate absorption and excretion of bile salt and other organic anions. Activation of FXR may play a potential therapeutic role in treatment of such diseases like hyperbilirubinemia and cholestasis. S-ademetionine (AdoMet) is an important biomolecule. It affects the intra-cellular gene expression, cell membrane liquidity needed for polyamine. It also, regulates growth, differentiation and function of cells through the synthetic action of transmethylation, transsulfation and polyamine. A number of studies showed that AdoMet can increase the bile acid, bilirubin and aminotransferase and decrease hepatic fibrosis development in patients with cholestasis-related hepatic disease [6,7]. However, AdoMet can be effective through a number of channels. Therefore, its molecular mechanisms that protect liver of hepatopathology include increasing the glutathione level in mitochondria, maintaining the dynamic state and stability of mitochondrial membrane and suppressing synthetic collagen of hepatic stellate cells $[6,8,9]$. These findings confirm that AdoMet

Correspondence to: Mao H, Department of Gastroenterology, Zhujiang Hospital, Southern Medical University, Guangzhou 510282, China, E-mail: huam@fimmu.com

Received: February 23, 2019, Accepted: March 23, 2019, Published: April 10, 2019

Citation: Mao H (2019) Hepatoprotective Effect of S-ademetionine in the treatment of Intrahepatic Cholestasis through Farnesoid X Receptor Mechanism in Rats. J Biomol Res Ther, 8: 176. doi: 10.35248/2167-7956.19.8.176

Copyright: $\odot 2019$ HMao H, et al. This is an open access article distributed under the term of the Creative Commons Attribution License, which permits unrestricted use, distribution, and reproduction in any medium, provided the original work is properly cited. 
could increase the expression of FXR and its target genes to promote excretion of bile acid thus protecting hepatic cells. Based on the above facts, this study was planned to investigate the possible hepatoprotective role of AdoMet to treat IHC liver in SD rats and an attempt to see if there is possible role of FXR in this mechanism.

\section{MATERIALS AND METHODS}

\section{Materials}

S-ademetionine for injection (Shanghai Abbott lab., China), GW4064 (Tocris Bioscience, Britain), Olive oil, $\alpha$-naphthyl isothiocyanate (Sigma Aldrich, Ltd., USA), dimethyl sulfoxide (DMSO) (Sigma Aldrich, Ltd., America USA); Beckman UniCel DxC Synchron 800 automatic biochemical analysis system (USA); Bio-Rad CFX96 Real-Time PCR System (Bio-Rad Company, USA); The oligonucleotide probe of Bsep, Mrp2 and Ntcp was synthesized by Guangzhou Biolink Biotechnology Co, Ltd.

\section{Animals}

Then, add $3 \mathrm{ml} \mathrm{11 \%} \mathrm{glutaraldehyde} \mathrm{and} \mathrm{leave} \mathrm{on} \mathrm{stirring} \mathrm{overnight.}$ Thirty six SD male rats weighing 100-120 g (SPF level) were purchased from Animal Experiment Center of Southern Medical University. During the test, animals were fed and sampled in accordance with the relevant regulations about laboratory animal management and protection of Southern Medical University.

\section{GROUPS, MODELING \& DRUG ADMINISTRATION}

These SD rats were divided into 6 groups, and were fed on a full diet standard diet for animals during tests, purchased from Southern Medical University for 1 week. These animals had unrestricted access to food and water and were housed in temperature- and humidity-controlled rooms and kept on a 12-h light/dark cycle. $36 \mathrm{SD}$ rats were housed in 6 cages with 6 in each cage. The room temperature of the laboratory was maintained at around $25^{\circ} \mathrm{C}$. After being fed adaptively for one week, they were randomly divided into groups A-F with 6 in each group. The rats in all groups were fasted for 12 hours with free access to water. After that, rats in group A were given olive oil of an equivalent amount (namely $5 \mathrm{ml} / \mathrm{kg}$ ) intragastrically. In the remaining five groups, IHC rat model was developed by giving 1\% ANIT olive oil mixed liquor intragastrically $[10,11]$. Normal diet was resumed 12 hours after intragastric administration. Subsequently, rats in each group received intraperitoneal injection of AdoMet solvent, Normal Saline (NS), AdoMet, GW4064, DMSO (GW4064 solvent) and AdoMet solvent for 2 consecutive days. Blood and liver tissue were taken from rats of all groups 2 days after intraperitoneal injection and 12 hours after the second dose separately. Each rat was anaesthetized with $6 \%$ chloral hydrate, and the abdomen cavity was cut open after sterile disinfection, abdominal aorta separated and 2 $\mathrm{ml}$ blood was withdrawn in a heparinized test tube. Subsequently, it was centrifuged at $4000 \mathrm{r} / \mathrm{min}$ for 10 minutes; plasma was separated and used for further analysis. Liver tissue were excised using sharp scissors, washed with normal saline, transferred into the micro tubes and stored in liquid nitrogen under $-80^{\circ} \mathrm{C}$ for analysis.

\section{Blood biochemical index test}

Beckman UniCel DxC Synchron 800 automatic biochemical analysis system was used to quantify test TBA, TBil, ALT, AST, ALP,
GGT. The manufactures protocol was followed while performing these tests.

Histopathology (HE staining): Liver tissues of all samples from all groups were fixed with $4 \%$ paraformaldehyde, and subsequently underwent steps like dehydration, embedment, Xylene dewaxing, slicing and haematoxylin-eosin staining. These slides were then observed microscopically for the degree of necrosis, inflammation, fibrosis and other structural changes.

Quantitative PCR (Q-PCR): The RNA was extracted from liver tissues using the TRIzol reagent (This manufacturing process is conducted as per the Instructions of the TRIzol kit to create $20 \mu \mathrm{l}$ reaction system). After extraction, the RNA concentration purity was checked spectrophotometrically. This was followed by the reverse transcription to synthesize cDNA. cDNA was used as the template to conduct expansion on the Quantitative PCR instrument (Bio-Rad CFX96 Real-Time PCR System). These were the reaction conditions for cDNA amplification: $95^{\circ} \mathrm{C}$ pre-denaturation for 1 min, $95^{\circ} \mathrm{C}$ denaturation for $10 \mathrm{sec}, 60^{\circ} \mathrm{C}$ annealing for $20 \mathrm{sec}, 40$ cycles in total. This is performed in triplicates. This is performed in triplicates. $\beta$-actin was used as the internal reference; the $2-\Delta \Delta \mathrm{Ct}$ method was used to conduct quantitative analysis.

Test of hybridization In situ: Five regions were randomly selected under 200-powered microscope, and the percentage of positive cells in each field was calculated. Judgments were made based on the percentage and color of positive cells. (1) Points scored according to the color of cells in the section: 0-colorless; 1-light brown; 2-brown; and 3-dark brown; (2) points scored according to the percentage positive cells accounted for among the total number of cells: $1-<30 \% ; 2-30 \% \sim 70 \%$; and $3->70 \%$. The total score equaled the product of the points scored in (1) and (2), and negative $(-)$ was defined as a score of 0 to 1 , weak positive $(+)$ as a score of 2 to 3 , and positive $(++)$ as a score of 4 or above.

\section{Statistical methods}

The statistical analysis was performed using the SPSS13.0 software. ANOVA or Chi-square test was used to analyze each group data. The statistical significance was noted if the P-value was less than 0.05 .

\section{RESULTS}

\section{General condition of IHC rats}

Healthy rats were vigorous, with glossy, soft and smooth hair good appetite, excreted clear urine, liver tissues had ruddy surface and had clear-cut margin. The rats which were given ANIT were listless, with lackluster hair, lower appetite, excreted deep yellow urine; dim surface and blunt margin of liver tissue were reported.

\section{AdoMet's protection of liver function}

The comparison between IHC+NS group and IHC+AdoMet solvent group/IHC+DMSO group did not show any statistically significant differences in terms of serum direct levels $(p>0.05$ for all) Table 1. When compared between IHC+AdoMet group and IHC+NS group/IHC+GW4064 group, the serum direct levels were statistically different ( $p<0.05$ for all), indicating that AdoMet can protect the liver of rats with ANIT-Induced Cholestasis (IHC) and has better liver protection than GW4064 of dosage used in 
the test Figure 1. When comparing IHC+ AdoMet group and olive oil + AdoMet solvent group, we did not find any statistical differences $(p>0.05)$, suggesting that the serological indicators of the cholestasis rats treated with AdoMet were close to those of the rats without cholestasis, and that AdoMet can satisfactorily restore the liver function in cholestasis rats Figure 2.

Table 1: Comparisons of the serum direct levels of group olive oil+SAMe solvent, group IHC+SAMe, group IHC+ GW4064 and group IHC+NS (chol, mmol/L.TBA,TBil, DBil, IBil, $\mu \mathrm{mol} /$ L.ALT, AST, ALT, GGT, IU/L.)

\begin{tabular}{|c|c|c|c|c|}
\hline & Olive oil +AdoMet solvent group & $\mathrm{IHC}+\mathrm{SAMe}$ & IHC+ GW4064 & $\mathrm{IHC}+\mathrm{NS}$ \\
\hline chol & $2.01 \pm 0.19^{\wedge}$ & $3.46 \pm 0.69^{\wedge} \cdot$ & $4.29 \pm 0.65$ & $4.95 \pm 1.78$ \\
\hline TBA & $48.27 \pm 38.23^{\Delta \bullet}$ & $41.80 \pm 4.32^{\wedge}$ & $281.33 \pm 118.20$ & $332.67 \pm 244.79$ \\
\hline DBil & $0.60 \pm 0.10^{\Delta \cdot}$ & $0.43 \pm 0.21^{\wedge}$ & $67.33 \pm 8.02$ & $71.57 \pm 25.25$ \\
\hline IBil & $2.20 \pm 0.10^{\Delta}$ & $5.03 \pm 4.06^{\boldsymbol{\Lambda}}$ & $56.83 \pm 14.45$ & $68.40 \pm 27.80$ \\
\hline AST & $149.00 \pm 16.00^{\Delta}$ & $118.67 \pm 17.21^{\wedge}$ & $987.00 \pm 643.47$ & $2600.33 \pm 146.77$ \\
\hline ALP & $694.00 \pm 28.60^{4}$ & $755.33 \pm 97.78^{\wedge}$ & $1038.67 \pm 160.18$ & $1352.00 \pm 46.52$ \\
\hline GGT & $1.00 \pm 0.60^{\Delta} \cdot$ & $3.00 \pm 1.65^{\Delta \cdot}$ & $6.33 \pm 1.16$ & $6.00 \pm 1.57$ \\
\hline
\end{tabular}

Note: ${ }^{\boldsymbol{\Delta}}$ as compared with IHC+NS group, $p<0.05 ;$ ' as compared with IHC+GW4064 group, $p<0.05$.

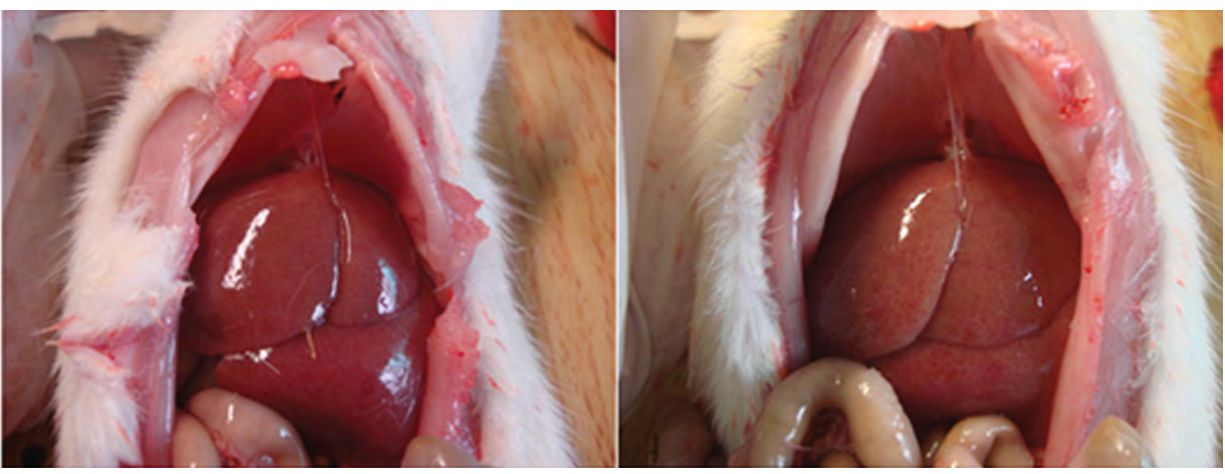

Figure 1: Liver shape and appearance comparison between normal healthy rat and IHC rat (left picture: liver of a normal/ healthy rat, right picture liver of an IHC rat).
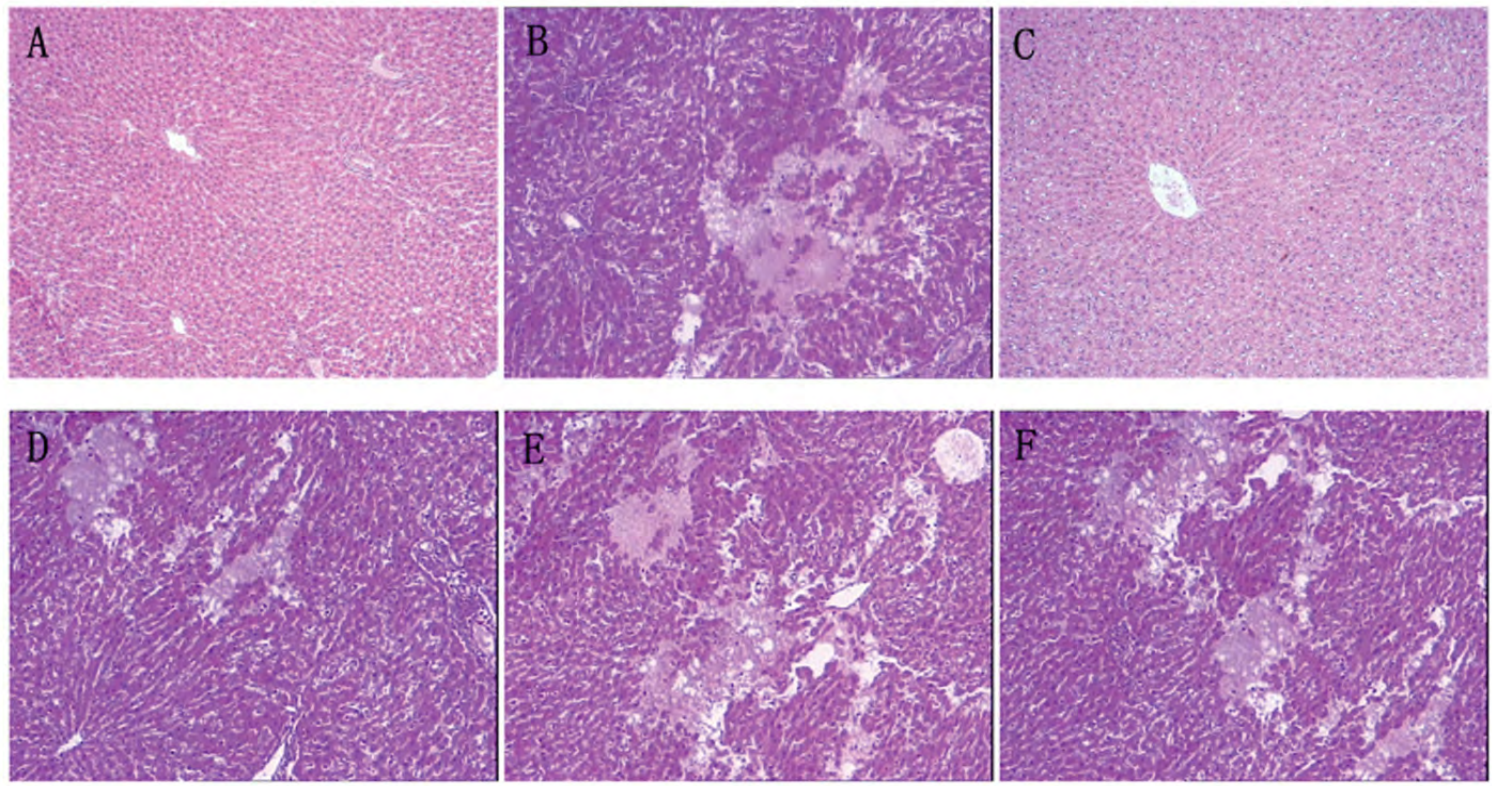

Figure 2: Comparison of histopathology of liver tissue of each group $(\times 100$ times $)$.

A: The olive oil +AdoMet solvent group, the liver tissue structure appeared normal, lobuli hepatis existed, and hepatic cords were seen in a radial pattern; B: The IHC+NS group, lobuli hepatis structure was disordered, and necrosis of flaky liver cells was visible; C: The IHC+AdoMet group, the lobuli hepatis structures existed, the hepatic cords were in order, and vacuole-shape changes were visible in hepatic cells; D: The IHC+GW4064 group, some lobuli hepatis structures were disordered, and necrosis of small flaky hepatic cells was visible; E: The IHC+DMSO group, the lobuli hepatis structure was in disorder, and necrosis of flaky liver cells were visible; F: The IHC+AdoMet solvent group, the lobuli hepatis structure was in disorder, and necrosis of flaky liver cells were visible. 
Relationship between AdoMet, FXR mRNA of liver tissue and Bsep, Mrp2 and Ntcp expression

FXR mRNA quantitation of liver tissue: In the comparison between $\mathrm{IHC}+\mathrm{NS}$ and $\mathrm{IHC}+$ AdoMet solvent group/IHC+DMSO group, there were no statistical differences in terms of the FXR mRNA expression quantity $(\mathrm{P}>0.05)$, which showed that the AdoMet solvent and DMSO had no significant impact on the expression quantity of FXR mRNA, and the AdoMet solvent and DMSO's interference with the FXR mRNA expression in the test could be ruled out. Comparison between IHC+ AdoMet, /IHC+GW4064, and $\mathrm{IHC}+\mathrm{NS}$ groups showed statistically significant differences

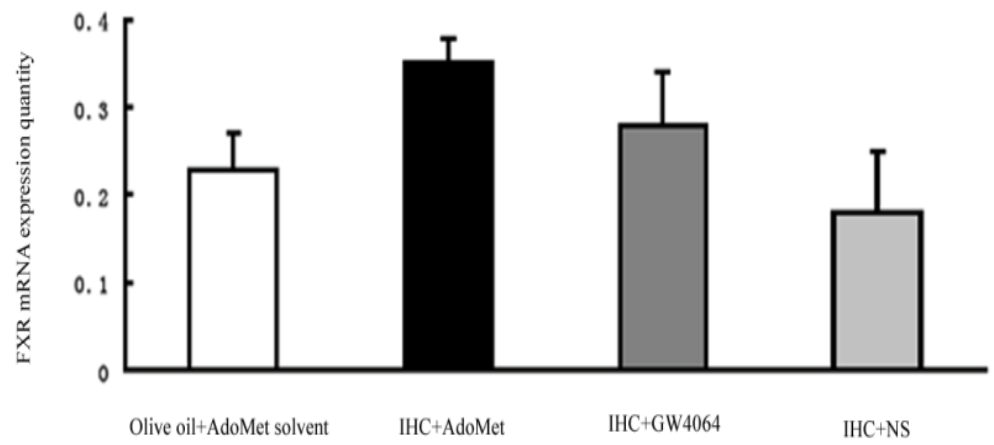

Figure 3: Comparison of FXR mRNA expression of group olive oil + SAMe solvent, group IHC+ SAMe, group IHC+ GW4064, and group IHC+NS.

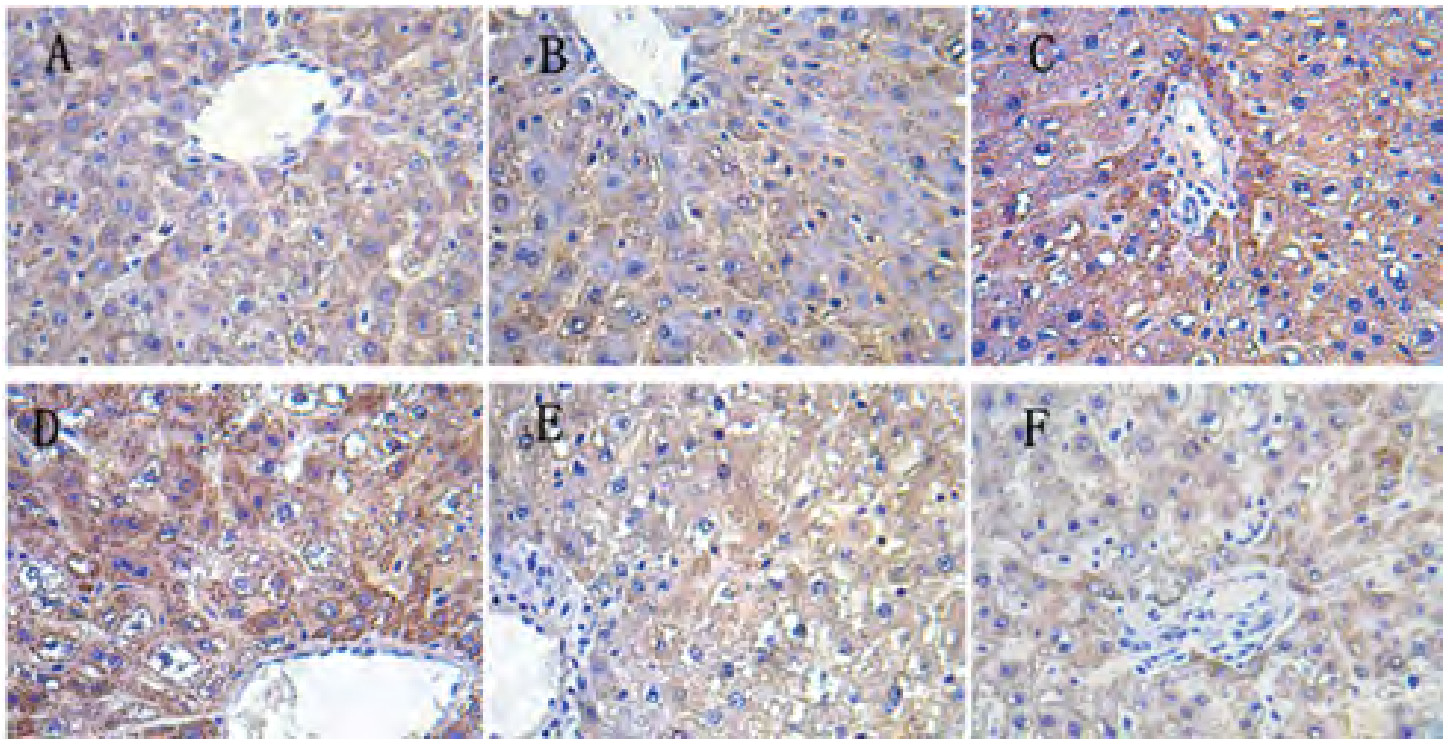

Figure 4: Comparison of Bsep expression of groups ( $\times 400$ times). AdoMet solvent group; B: IHC+NS group; C:IHC+ AdoMet group; D: IHC+GW4064 group; E: IHC+DMSO group; F: IHC+AdoMet solvent group.
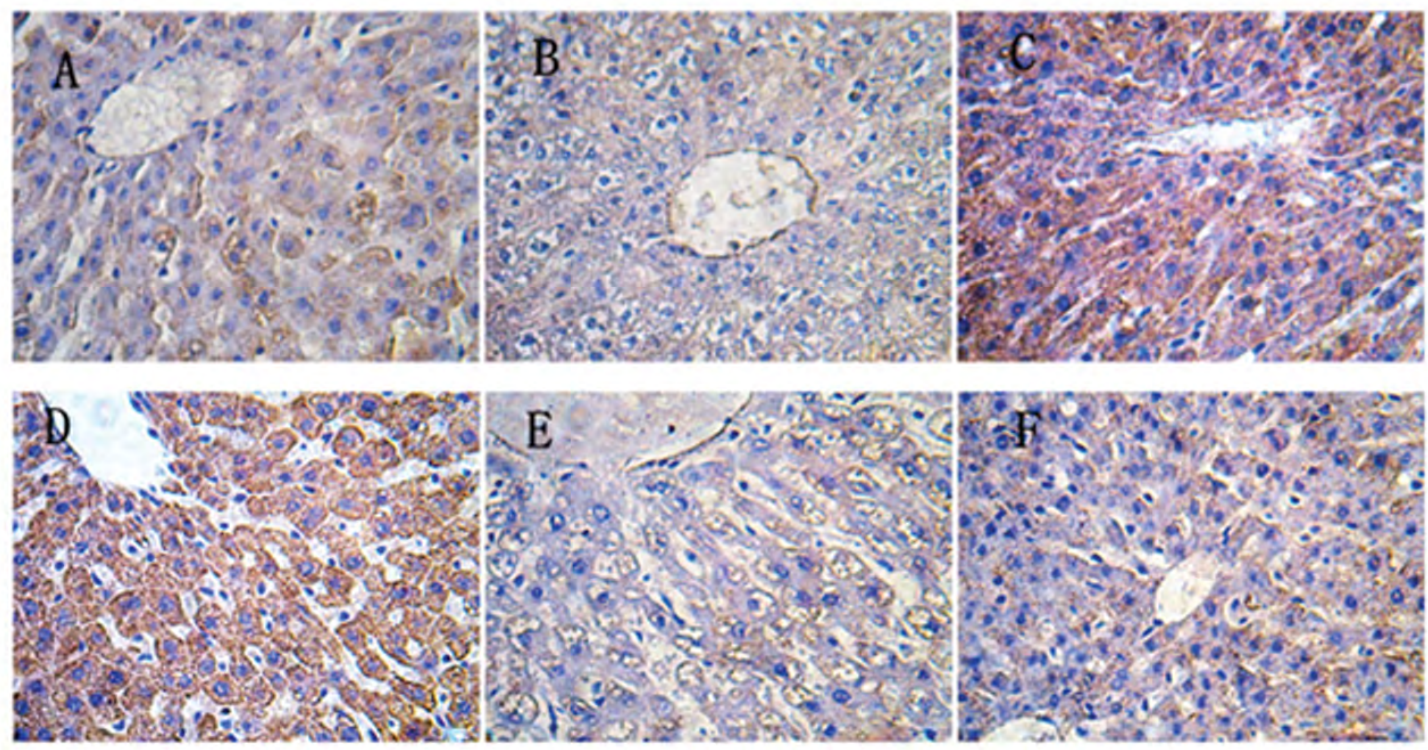

Figure 5: Comparison of Mrp2 expression of groups ( $\times 400$ times).

A: Olive oil + AdoMet solvent group; B: IHC+NS group; C: IHC+ AdoMet group; D: IHC+GW4064 group; E: IHC+DMSO group; F: IHC+AdoMet solvent group. 


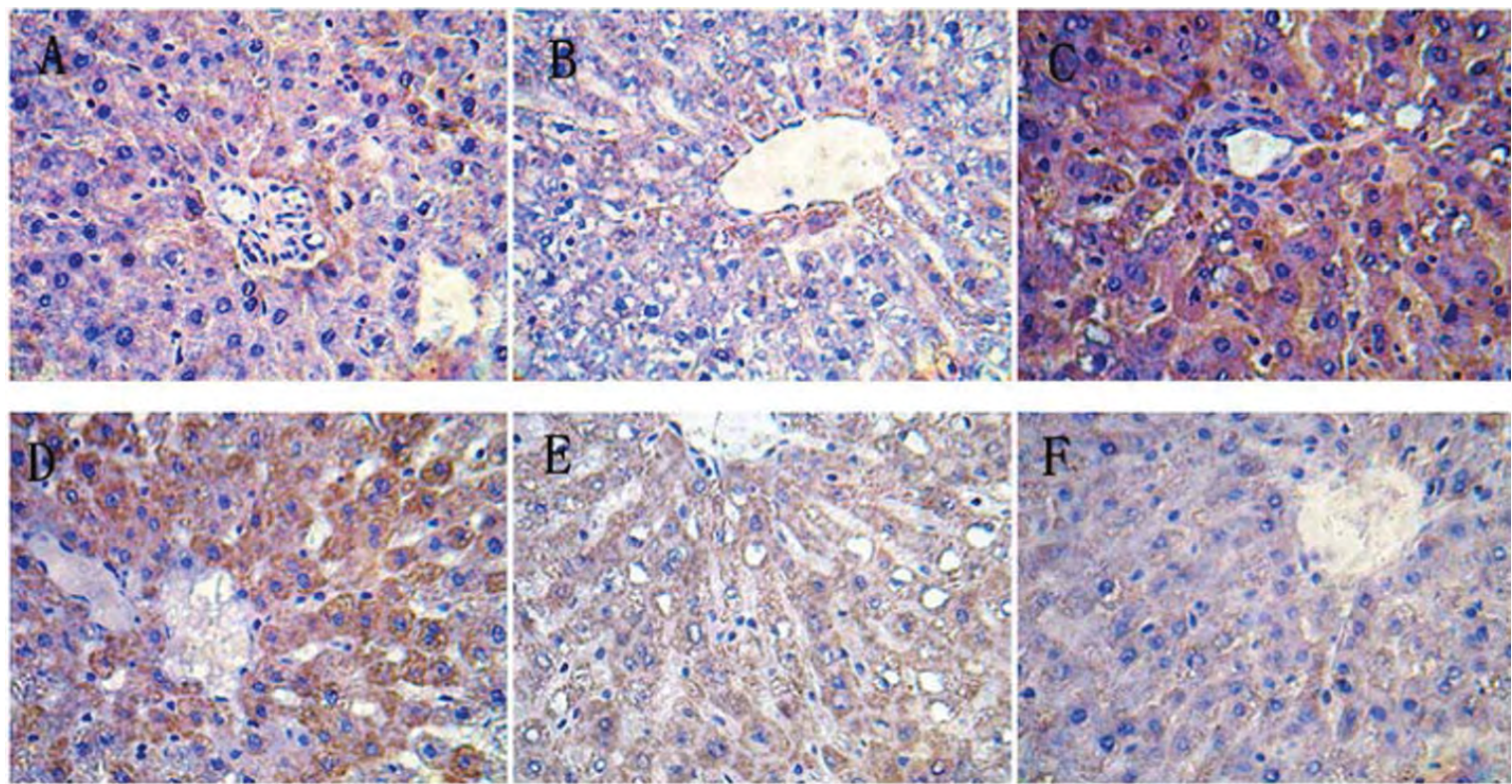

Figure 6: Comparison of Ntcp expression of groups ( $\times 400$ times).

A: Olive oil +AdoMet solvent group; B: IHC+NS group; C: IHC+AdoMet group; D: IHC+GW4064 group; E: IHC+DMSO group; F: IHC+AdoMet solvent group.

Table 2: Semi quantitative analysis of Bsep, Mrp2, Ntcp expression in liver tissues among various groups.

\begin{tabular}{clllll}
\hline Group & $\mathbf{n}$ & - & + & ++ & Positive rate $^{*}(\%)$ \\
\hline Olive oil +AdoMet solvent group & 6 & 5 & 1 & 0 & 16.7 \\
IHC+NS group & 6 & 5 & 1 & 0 & 16.7 \\
IHC+ AdoMet group $\boldsymbol{\Delta}$ & 6 & 0 & 2 & 4 & 100 \\
IHC+GW4064 group $\boldsymbol{\Delta}$ & 6 & 0 & 2 & 4 & 100 \\
IHC+AdoMet solvent group & 6 & 5 & 1 & 0 & 16.7 \\
IHC+DMSO group & 6 & 5 & 1 & 0 & 16.7 \\
\hline
\end{tabular}

$(\mathrm{P}<0.05)$. The used doses of AdoMet and GW4064 may increase the expression of FXR mRNA in the liver tissues [3]. There were no statistically meaningful differences between the IHC+ AdoMet group and $\mathrm{IHC}+\mathrm{GW} 4064$ group $(\mathrm{P}>0.05)$, and the impact on the expression did not vary much between the two groups. The comparison between the IHC+ AdoMet and olive oil + AdoMet solvent groups showed that statistical differences exist $(\mathrm{P}<0.05)$. The expression in the IHC+ AdoMet group increased compared with the olive oil + AdoMet solvent group, which indicated that AdoMet's liver protection could related to the increase in the FXR mRNA expression Figure 3.

\section{Bsep, Mrp2, Ntcp expression of liver tissue}

Compared with the IHC+AdoMet solvent and IHC+DMSO group, the positive rates of the Bsep, Mrp2, Ntcp expression had no distinct differences (all were 16.7\%), and the impact of the AdoMet solvent and DMSO on the Bsep expression was ruled out. When compared with the olive oil + AdoMet solvent /IHC+NS group/IHC+AdoMet solvent group/IHC+DMSO group, the positive rates of the Bsep expression in the $\mathrm{IHC}+$ AdoMet group and $\mathrm{IHC}+\mathrm{GW} 4064$ group were enhanced $(\chi 2=5.486, \mathrm{P}=0.019)$ and was mainly expressed in cytoplasm Figures 4-6 and Table 2.

\section{DISCUSSION}

AdoMet is an important biomolecule which plays an important role in various biochemical reactions $[12,13]$.
Relevant studies showed that only supplementing methionine exogenously for patients with hepatocirrhosis cannot increase the level of AdoMet in vivo such that even if methionine levels are as high as 7 times, it did not raise the AdoMet levels significantly, rather excessive methionine may also lead to toxic reactions [14]. Therefore, when pathological changes of the liver which causes insufficient synthesis of AdoMet occure, we should avoid the process where methionine synthetizes AdoMet, and instead supplement AdoMet therapeutically to make up for the insufficient levels in vivo. Knowing the critical role of the AdoMet physiologically, it is considered an important therapeutics choice to be used to recover the liver damage casued e.g IHC. Therefore, in the present study we investigated if AdoMet can protect the ANIT induced IHC in rats and with a possible FXR related mechanism.

AdoMet's protection of liver of IHC rat

In case of liver damage, the expression of cells, adhesion molecules and active oxygen free radicals increases, which aggravates damage to hepatic cells and tissues. Hepatic cells, however, generate antioxidases, such as glutathion peroxidase and glutathione reductase, as the endogenous oxygen radical scavengers to protect cells from damage [15]. Specifically, GSH is an important oxygen radical scavenger in cells. S-adenosylhomocysteine is the product of AdoMet methylation. It is converted into homocysteine and then generates cysteine under the effect of transsulfation, and is synthesized into GSH by cysteine. Effectively, increasing the GSH level in mitochondria of hepatic cells may resist the damage to cells caused by oxygen radicals and inflammatory substances, 
and thus maintain the liquidity of mitochondrial membrane, and the normal function and detoxication of mitochondria and cells $[11,16]$. AdoMet can not only prevent ROS's damage to hepatic cells, but also reduce the damage to hepatic cells by regulating the balance between proinflammatory cytokine and anti-inflammatory cytokine [11]. Relevant studies showed that AdoMet may reduce liver damage mediated by the pro-inflammatory cytokine TNF- $\alpha$ by down-regulating the nuclear factor-kappa B (NF-кB); GSH synthesized by AdoMetAdoMet may also reduce the expression of TNF- $\alpha .17$ Meanwhile, the induced anti-inflammatory cytokine IL-10 protects the liver $[11,17,18]$. In addition, AdoMet may act as the methyl donor to offer methyl to increase of the proportion of phosphatidylcholine/phosphatidyl ethanolamine may increase the liquidity of membrane and activity of the $\mathrm{Na}^{+}$- $\mathrm{K}+$ - ATP enzyme, which reduce stickiness of the cell membrane, speed up operation of bile acid and excretion of metabolites, and ultimately affects transduction of cell signals [19-22]. AdoMet also plays an important role in maintaining the normal patterns of fibrils and microtubules and actin content of the damaged hepatic cells in rats in order to resist the liver damage caused by cholestasis $[23,24]$.

\section{CONCLUSION}

AdoMet may protect hepatic cells in the damaged liver by resisting oxygen radicals, regulating the balance between pro-inflammatory cytokines and anti-inflammatory cytokines, suppressing apoptosis, and increasing the liquidity of cell membranes. The results of this study showed that in the serum of the IHC rats treated with AdoMet, the TBA, TBil, ALT, AST, ALP and GGT levels were significantly lower than those of the IHC rats treated with NS, that hepatic histological damage is lower than the liver tissue damage in IHC rats, and there are no distinct differences in terms of serological indicators and liver tissue damage compared with the rats with no IHC. This indicates that AdoMet can protect the liver of ANITinduced IHC rats, and restore the liver function of such cats to the normal level [25]. For the IHC rats treated with GW4064, the TBA, TBil, ALT, AST, ALP and GGT levels in the serum and the pathologic changes in the liver tissues were compared to those with no IHC, and it can be concluded that GW4064 has potential to protect the liver to some extent. As mentioned above, exogenous AdoMet may recover the liver damage caused by insufficient AdoMet in vivo, which complies with the test result where AdoMet was given to cholestasis rats and which effectively restored the liver function. This probably indicates that AdoMet may be used to treat and effectively prevent IHC-related hepatic damage.

AdoMet's protection of Liver \& FXR-related mechanism

Relevant existing studies showed that FXR suppresses bile acid synthesis by decreasing bile acid synthetase, mainly synthesis of CYP7A1 and may directly regulate and encode the bile component transporters Bsep, Mrp2 and Ntcp to regulate metabolism of bile acid. When there are transporter synthesis disorders, the bile transport is reduced, and bile accumulates in vivo, which results into the inflammatory reaction of hepatic cells, fibrosis, hepatocirrhosis, liver cancer or cholangiocarcinoma is developed further [26]. Bsep is the important transport protein for bile acid excretion. When the gene with the code Bsep mutates, will leading to progressive familial intrahepatic cholestasis [27]. The defect of the gene with the code Mrp2 in vivo may cause the Dubin-Johnson syndrome, familial chronic nonhemolytic jaundice. In the body of a cholestasis rat, the Ntcp level is reduced, the level of toxic bile acid is increased, which also indicates that the reabsorption and recirculation of bile acid are related to cholestasis. This shows that these transporters play an important role in hepatic cells removal of bile components and toxic metabolites. Therefore, increasing the Bsep, Mrp2 and Ntcp level and function of FXR and transcriptive synthesis in hepatic cells is the priority in promoting bile excretion and metabolism, and the key to treating cholestasis. The expression of FXR is regulated by various factors at the transcription level. Specifically, various inflammatory factors and transcription factors may directly or indirectly control the expression of FXR. TNF- $\alpha$ and IL-1 almost completely block the FXR reaction element promotor, which significantly reduces the FXR expression. IFN- $\gamma$ may also induce the signal transducers and activator of transcription (STAT) to suppress the transcription and expression of the FXR gene [28]. NF- $\kappa B$ is an inflammation regulation factor. It reduced the expression of FXR mRNA in the inflammatory reaction of the liver, suppressed the gene expression mediated by FXR, and antagonized FXR. After NF- $\mathrm{KB}$ is activated, participates in the transcription of such inflammation media as TNF- $\alpha$, IL- 1 and IFN- $\gamma$, and further suppresses the expression of FXR mRNA [29]. In conclusion, the inflammatory factors TNF- $\alpha$, IL-1, NF- $\kappa$ B and IFN- $\gamma$ may directly or indirectly suppress the expression of FXR. This study showed that suppressing inflammatory factors and regulating FXR are an effective factor for liver protection. AdoMet may suppress the expression of TNF- $\alpha$ l, NF- $\kappa$ B, IL-1 and IFN$\gamma$ in case of liver damage, protects the liver from being harmed by inflammatory factors, reduces the inhibitory effects of TNF- $\alpha$, NF- $\kappa$ B, IL- 1 and IFN- $\gamma$ on FXR of the liver tissue, thus increases the expression of FXR, regulates the transporter expression on the liver cell membrane, promotes bile excretion and metabolism, and protects the liver. The rats with cholestasis treated with AdoMet, the expression of FXR mRNA and positive expression of Bsep, Mrp2 and Ntcp in the liver tissues were higher than those with no cholestasis. This indicates that AdoMet could be through the above mechanism of action, and is related to the increased expression of FXR mRNA in the liver tissues. This is probably related to the inhibitory effects of the increased inflammatory factors TNF- $\alpha$, NF$\kappa \mathrm{B}$, IL-1 and IFN- $\gamma$ on FXR while AdoMet reduces the damage of hepatic cells. In conclusion, AdoMet's liver protection is likely to be related to the existing mechanisms, and to the reduced suppression or direct effects of the inflammatory factors TNF- $\alpha$, NF- $\mathrm{kB}$, IL-1 and IFN- $\gamma$ on FXR which increases the FXR level, synthesizes the bile component transporter and promotes bile excretion and metabolism. It may also be related to the positioning of increased bile component transporters in the cell membrane.

\section{ACKNOWLEDGEMENTS}

We sincerely acknowledge the intellectual support of Professor Linlang Guo and Dr. Yufa Li, Department of Pathology, Zhujiang Hospital of Southern Medical University, Guangzhou, China, while performing this study.

\section{REFERENCES}

1. Tu H, Okamoto AY, Shan B. FXR, a bile acid receptor and biological sensor. Trends Cardiovasc Med. 2000;10:30-35.

2. Plass JR, Mol O, Heegsma J, Geuken M, Faber KN. Farnesoid X receptor and bile salts are involved in transcriptional regulation of the gene encoding the human bile salt export pump. Hepatology. 2002;35: 589-596.

3. König J, Nies AT, Cui Y, Leier I, Keppler D. Conjugate export pumps of the multidrug resistance protein (MRP) family: localization, substrate 
specificity and MRP2-mediated drug resistance. Biochim Biophys Acta. 1999; 1461:377-394.

4. Fardel O, Jigorel E, Le Vee M, Payen L. Physiological, pharmacological and clinical features of the multidrug resistance protein 2. Biomed Pharmacother. 2005;59:104-114.

5. Borst $\mathrm{P}$, Elferink RO. Mammalian $A B C$ transporters in health and disease. Annu Rev Biochem. 2002; 71:537-592.

6. Yang H, Ramani K, Xia M, Ko KS, Li TW. Dysregulation of glutathione synthesis during cholestasis in mice: molecular mechanisms and therapeutic implications. Hepatology. 2009;49:1982-1991.

7. Caballero F, Fernández A, Matías N, Martínez L, Fucho R. Specific contribution of methionine and choline in nutritional nonalcoholic steatohepatitis: impact on mitochondrial S-adenosyl-L-methionine and glutathione. J Biol Chem. 2010;285:18528-18536.

8. Andringa KK, King AL, Eccleston HB, Mantena SK, Landar A. Analysis of the liver mitochondrial proteome in response to ethanol and S-adenosylmethionine treatments: novel molecular targets of disease and hepatoprotection. Am J Physiol Gastrointest Liver Physiol. 2010;298:G732-G745.

9. Akwabi-Ameyaw A, Bass JY, Caldwell RD, Caravella JA, Chen L. Conformationally constrained farnesoid X receptor (FXR) agonists: Naphthoic acid-based analogs of GW 4064. Bioorg Med Chem Lett. 2008;18:4339-4343.

10.Liu Y, Binz J, Numerick MJ, Dennis S, Luo G. Hepatoprotection by the farnesoid X receptor agonist GW4064 in rat models of intra- and extrahepatic cholestasis. J Clin Invest. 2003;112:1678-1687.

11. Purohit V, Abdelmalek MF, Barve S, Benevenga NJ, Halsted CH. Role of S-adenosylmethionine, folate, and betaine in the treatment of alcoholic liver disease: summary of a symposium. Am J Clin Nutr. 2007;86:14-24.

12.Lu SC, Mato JM. S-adenosylmethionine in liver health, injury, and cancer. Physiol Rev. 2012;92: 1515-1542.

13. Mato JM, Martínez-Chantar ML, Lu SC. S-adenosylmethionine metabolism and liver disease. Ann Hepatol. 2013;12:183-189.

14. Yang YJ, Huang F. Role of hepatic stellate cells and correlated cytokines in the formation of hepatic fibrosis. W Chinese J of Digestol. 2007; 15:2885-2890.

15.Colell A, García-Ruiz C, Morales A, Ballesta A, Ookhtens M. Transport of reduced glutathione in hepatic mitochondria and mitoplasts from ethanol-treated rats: effect of membrane physical properties and S-adenosyl-L-methionine. Hepatology. 1997;26:699-708.

16. García-Ruiz C, Morales A, Colell A, Ballesta A, Rodés J. Feeding S-adenosyl-L-methionine attenuates both ethanol-induced depletion of mitochondrial glutathione and mitochondrial dysfunction in periportal and perivenous rat hepatocytes. Hepatology. 1995;21:207-214.

17. Colell A, García-Ruiz C, Miranda M, Ardite E, Marí M. Selective glutathione depletion of mitochondria by ethanol sensitizes hepatocytes to tumor necrosis factor. Gastroenterology. 1998;115: 1541-1551.

18. Song Z, Uriarte S, Sahoo R, Chen T, Barve S. S-adenosylmethionine (SAMe) modulates interleukin-10 and interleukin-6, but not TNF, production via the adenosine (A2) receptor. Biochim Biophys Acta. 2005;1743:205-213.

19. Hirata F, Axelrod J. Phospholipid methylation and biological signal transmission. Sci. 1980;209:1082-1090.

20.Hirata F, Toyoshima S, Axelrod J, Waxdal MJ. Phospholipid methylation: a biochemical signal modulating lymphocyte mitogenesis. Proc Natl Acad Sci USA. 1980;77:862-865.

21. Hirata F, Viveros OH, Diliberto EJ Jr, Axelrod J. Identification and properties of two methyltransferases in conversion of phosphatidylethanolamine to phosphatidylcholine. Proc Natl Acad Sci USA. 1978;75:1718-1721.

22.Crews FT. Effects of membrane fluidity on secretion and receptor stimulation. Psychopharmacol Bull. 1982;18:135-143.

23.Roman ID, Johnson GD, Coleman R. S-adenosyl-L-methionine prevents disruption of canalicular function and pericanalicular cytoskeleton integrity caused by cyclosporin A in isolated rat hepatocyte couplets. Hepatology. 1996;24:134-140.

24. Ramírez T, García-Montalvo V, Wise C, Cea-Olivares R, Poirier LA. S-adenosyl-L-methionine is able to reverse micronucleus formation induced by sodium arsenite and other cytoskeleton disrupting agents in cultured human cells. Mutat Res. 2003;528:61-74.

25.Di Padova C, Di Padova F, Tritapepe R, Stramentinoli G. S-adenosylL-methionine protection against alpha-naphthylisothiocyanate-induced cholestasis in the rat. Toxicol Lett. 1985;29:131-136.

26.Pandak WM, Li YC, Chiang JY, Studer EJ, Gurley EC. Regulation of cholesterol 7 alpha-hydroxylase mRNA and transcriptional activity by taurocholate and cholesterol in the chronic biliary diverted rat. J Biol Chem. 1991;266:3416-3421.

27. Strautnieks SS, Bull LN, Knisely AS, Kocoshis SA, Dahl N. A gene encoding a liver-specific $\mathrm{ABC}$ transporter is mutated in progressive familial intrahepatic cholestasis. Nat Genet. 1988;20:233-238.

28. Renga B, Migliorati M, Mencarelli A, Fiorucci S. Reciprocal regulation of the bile acid-activated receptor FXR and the interferon-gamma-STAT-1 pathway in macrophages. Biochim Biophys Acta. 2009;1792:564-573.

29. Wang YD, Chen WD, Wang M, Yu D, Forman BM. Farnesoid X receptor antagonizes nuclear factor kappaB in hepatic inflammatory response. Hepatology. 2008;48:1632-1643. 